\title{
Somatic Cell Variation during Uninterrupted Growth of Neurospora crassa in Continuous Growth Tubes
}

\author{
By H. BERTRAND, K. J. McDOUGALL AND T. H. PITTENGER \\ Department of Agronomy, Kansas State University, \\ Manhattan, Kansas, U.S.A.
}

(Accepted for publication II August 1967)

\begin{abstract}
SUMMARY
Continuous growth of Neurospora crassa was studied to determine whether the organism was capable of uninterrupted hyphal elongation, whether there was any evidence of homeostatic mechanisms controlling growth over extended periods and, particularly, whether spontaneous mutants with suppressive phenotypes were capable of being expressed. Parallel cultures, $A$ and $B$, having a common origin, were incapable of uninterrupted growth but permanent cessations of growth were not encountered. The A-culture showed frequent stops of long duration whereas B had infrequent stops of short duration. This stop-start growth behaviour in the A- and Bcultures was determined by two different cytoplasmic factors. Both extranuclear mutants show decreased cytochrome-c oxidase activity (Bertrand \& Pittenger, unpublished). These stopper phenotypes may be similar to 'vegetative death' in Aspergillus and 'senescence' in Podospora. Both A- and B-cultures showed several major increases and decreases in rate, in addition to more frequent minor fluctuations. The general constancy of growth, however, and the routine restoration of growth after cessations, as well as following either transient or long-term growth rate decreases, indicated that homeostatic mechanisms were capable of buffering the organisms against most deleterious intracellular effects. Two spontaneous nuclear mutants with decreased growth rates as homokaryons also accumulated during growth. These altered nuclear types, whose growth rates were clearly non-adaptive, nevertheless had selective values. Their proportions increased significantly during growth to the extent of either completely displacing the original type or of reaching a high enough proportion so that growth rates were decreased and the morphology of the cultures altered. These nuclear and extranuclear mutants appeared to have a selective advantage because of suppressive characteristics.
\end{abstract}

\section{INTRODUCTION}

The use of a continuous growth tube for the study of uninterrupted mycelial growth of Neurospora crassa is strictly a laboratory innovation since comparable conditions are never expected in nature. Under such controlled conditions in a rapidly growing organism such as Neurospora, where growth by hyphal elongation in a wild-type strain often exceeds $120 \mathrm{~mm}$./day, the hyphal tips are continually advancing on to fresh medium as they progress down a growth tube. Homeostatic mechanisms are expected to maintain a constant growth rate by buffering the organism against deleterious effects that might accrue from spontaneous mutations and unfavourable physiological conditions. Continuous growth in a constant environment thus provides an unusual opportunity to observe the efficiency of such mechanisms over extended

Vol. 50, No. 2, was issued 21 February 1968 
periods of vegetative growth. Such a study also provides a basis for comparing the effects of long-term growth in Neurospora with those observed in other fungi, such as Aspergillus and Podospora. In the latter, continuous hyphal elongation invariably results in a cytoplasmically determined irreversible cessation of growth described as 'vegetative death' (Jinks, I959) and 'senescence' (Marcou, I96I). In fact it was the results of such studies with Podospora and other slow-growing fungi which suggested that indefinite hyphal propagation is impossible in those fungi (Marcou, 196I). And lastly, such a study should enable the investigator, through systematic sampling and analyses of asexual spores of the culture, to determine whether the somatic cell variation expected to occur during the continuous asexual growth of such cultures has a genetic basis. Two observations in this regard by Jinks (I958, I959)-that cytoplasmic elements are involved in naturally occurring variation in aged cultures and our own that many cytoplasmic mutants are characterized by a stop-and-start growth phenotype recognized only in growth tubes-originally prompted the present work.

It is clear from a variety of experiments that the coenocytic nature of the mycelium provides conditions that would allow the accumulation of a variety of spontaneous recessive mutants, which would be rapidly eliminated in a population of uninucleate cells. It can be assumed that spontaneous mutations which occur during growth of the original homokaryotic culture would provide, with time, a heterokaryon and heteroplasmon composed of many genetically different types, and that the effect of the majority of such recessive mutants on the continuous growth of the culture would be negligible. Since Neurospora grows by hyphal elongation and branching, and since nuclear ratios in heterokaryons appear to be relatively stable, it appears that protoplasmic streaming and hyphal fusions should aid in continuous mixing and dilution of spontaneously occurring mutant nuclei and cytoplasmic determinants with their normal homologues within the mycelium. However, because of those factors, it would be expected that mutations which occur in one member of a large population of cellular organelles rarely would be expected to accumulate and be maintained in sufficient numbers to alter the phenotype of the entire culture. Consequently, it could be anticipated that genetically determined changes in the growth rate or morphology of a growing culture might occur only when the mutant type had unusual properties, as being either a dominant mutation or one that suppresses both the action and division of its normal counterpart. Since many of the extranuclear mutations in fungi have that property of suppressiveness which Jinks (1964) believes can be explained best on the basis of superior division rates of the mutant determinant, it was believed that continuous growth might provide both extranuclear and nuclear mutants with unique properties.

\section{METHODS}

Organisms. The strains of Neurospora crassa used were: ad-4al-2A (F4), adenine4; I5300, albino-2), pan-I al-I (553I, pantothenic-I ; 4637T, albino-I); nic-I al-288a and nic-Ial-255A (34I6, nicotinic-I; I5300, albino-2), and a standard wild-type strain, 74-OR-8- $I a$. All the strains were heterokaryotically compatible with one another, except for $74 a$ and the effect of mating type $a$. Except in cases where the complete genotype seems necessary for emphasis or clarity, the following abbreviations are used: $a d$, pan, nic, and $74 a$.

Conditions used for continual hyphal propagation. Special continuous growth tubes were designed to study the effects of perpetual hyphal propagation (McDougall \& 
Pittenger, 1962). The Pyrex growth tubes were $615 \mathrm{~mm}$. long with inside diameters of about $25 \mathrm{~mm}$. Four cotton-stoppered sampling ports $(8 \mathrm{~mm}$. diameter) were spaced along the tops of the tubes for aeration and to facilitate removal of conidia, which formed in abundance under the openings. Ends of the tubes had 24/40 standardtaper ground-glass interchanges. Closures made of the same type of glass joints were used for sealing the ends which were not joined to other tubes. Each $615 \mathrm{~mm}$. section was filled with $100 \mathrm{ml}$. medium.

An $a d-4 a l-2 A$ strain was inoculated at the proximal end of the initial section. Before the culture reached the end of a section, the distal closure was removed and another section filled with medium joined to the first one. Once the mycelium was well established in the new section, the tubes were separated and sealed with sterile closures. Subcultures were prepared from masses of aerial hyphae and conidia removed from at least one sampling port before the proximal section was discarded. The mature subcultures in slopes were stored at $4^{\circ}$. Repeated additions of sections permitted uninterrupted growth by hyphal elongation for more than 4 years with no need to subculture the organism.

The main culture was called the A-culture and sections of the corresponding growth tube were numbered from $\mathrm{A}_{\mathrm{I}}$ to $\mathrm{A}_{4} \mathrm{I}$. Because of a significant change in growth rate, a parallel culture, the B-culture, was started from the proximal end of section A I6, in addition to continuing the A-culture from the distal end of the same section. The sections of the growth tube corresponding to the B-culture were numbered B I6 to B I60.

The position of the frontier of the culture was marked daily on the growth tube. Daily average growth rates were determined from these markings.

Types of media used. Vogel medium ( 1956$)$ with $\mathrm{I} \cdot 5 \%(\mathrm{w} / \mathrm{v})$ sucrose as carbon source and solidified with $\mathrm{I} \cdot 5 \%$ (w/v) of unwashed Difco Bacto-Agar was used in all growth experiments. All experiments were made at $30^{\circ}$. Routine growth-rate determinations of subcultures or single conidial isolates were done in $500 \mathrm{~mm}$. growth tubes (Ryan, Beadle \& Tatum, 1943) containing $30 \mathrm{ml}$. medium. The medium of Westergaard \& Mitchell (1947) was used for crosses and for plating of conidia. Supplements at the following concentrations were used: adenine, $0.2 \mathrm{mg} . / \mathrm{ml}$., and nicotinamide and calcium pantothenate, $0.005 \mathrm{mg} . / \mathrm{ml}$. Complete medium was prepared by adding $0.25 \%(\mathrm{w} / \mathrm{v})$ Casamino acids and $0.25 \%(\mathrm{w} / \mathrm{v})$ yeast extract to basal medium containing a carbon source.

Methods used to plate conidia, to prepare heterokaryons, to recover homokaryotic conidial isolates from heterokaryons, and for their analyses can be found elsewhere (McDougall \& Pittenger, I966; Bertrand \& Pittenger, unpublished).

Selection of mutants for analysis. The only criteria used to select cytoplasmic and nuclear mutants for analysis during continuous growth were: (I) prominent changes in growth rate; (2) visible changes in morphology of the culture, such as those related to abundance of aerial growth, conidiation, and lysis of the culture; (3) changes in both sexual and asexual reproductive capacity; (4) changes in morphology of colonies formed when conidia were plated on sorbose medium. No attempt was made to determine the frequency of auxotrophic or visible mutants which may have accumulated during growth. Consequently, the genetic analysis was confined to changes in growth rate and morphological mutants first observed in the A- and B-cultures themselves, rather than analysis of somatic cells routinely isolated from these cultures. 


\section{RESULTS}

Space is not available nor would it be particularly meaningful to present a single growth curve for either the A- or B-cultures over a 4-year period which would include all the daily minor variations in growth, including times when growth progressively slowed and finally ceased temporarily. Instead, average maximum growth rates in each section were calculated and plotted against the sections of the continuous growth tube. Such calculations adequately picture the major increases and decreases, as shown in Fig. I. Because of the variable daily rates observed in A23 to A4I, the maximum $24 \mathrm{hr}$ rates or average maximum rates in these sections were used in calculating the data presented in Fig. I rather than the average of all daily rates. This procedure avoids minor fluctuations in rate preceding cessation of growth. It was adopted to express more accurately the potential growth rate of the culture in any particular section of the growth tube.

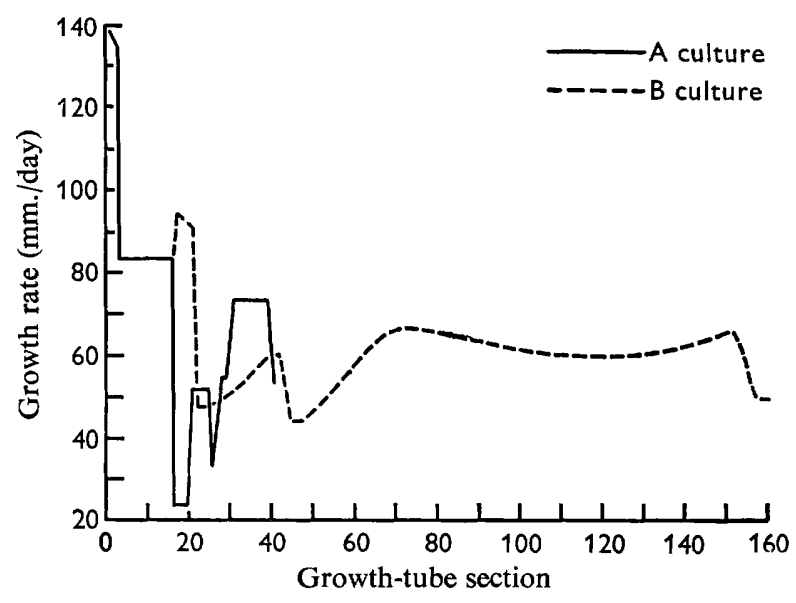

Fig. I. Growth rates of the adal-2 $A$ culture of Neurospora crassa during continuous hyphal elongation in growth tubes $\mathrm{A}$ and $\mathrm{B}$ over a 4-year period. For sections where growth was erratic, or stopped completely, the maximum $24 \mathrm{hr}$ growth rate, rather than the average growth rate, has been plotted to avoid minor fluctuations and to express more accurately the potential rate of the culture.

Although the data presented in Fig. I show several significant increases and decreases in the rate of growth of the A- and B-cultures, the most unusual feature, but not shown in the growth curve, was the fact that growth stopped completely 68 different times, 48 in the A-culture and 20 in the B-culture. The growth behaviour in the A- and B-cultures before stopping and after resuming is shown in some representative 'growth maps' in Table I, but no attempt was made to present such data for the entire period.

\section{Description of growth of the A-culture of strain adal-2A}

The ad al-2A culture grew $137 \mathrm{~mm}$./day through the first $1200 \mathrm{~mm}$. of the A-growth tube, which was surprising since the adal-2A strain normally grows at only 80-85 $\mathrm{mm}$./day. Although this initial rate of $5.7 \mathrm{~mm}$./hr was extremely high, even for a wildtype strain, it could not be repeated either with subcultures from sections A I and A2, 
single conidial isolates from those sections, or with cultures of the strain used to initiate section A I. Nevertheless, the high average rate was real, for it represented nearly 9 days of growth. It was assumed that factors determining the initial vigour either were not heritable and were eliminated during growth and conidial reproduction, or that the rate was affected by unspecified environmental conditions which could not be reproduced.

A significant change in growth rate was observed in A I6 (Fig. 1). Subsequent growth was slow and erratic until it ceased temporarily in A 23. By then the culture had grown nearly $14,200 \mathrm{~mm}$. in 305 days. After the stop in A 23, the culture grew in highly irregular cycles consisting of a period of active hyphal elongation followed by temporary cessation. For example, growth resumed in A 23 at $38 \mathrm{~mm}$./day after 8 days of no growth. However, 3 days later the culture again stopped, this time for 10 days, which in turn was followed by $3 \mathrm{I}$ days of active growth and ${ }_{5} 5$ days of no growth. The stop-start growth pattern was maintained until the culture was discarded in A4I when it became contaminated with bacteria, about 1065 days after it stopped for the first time. During this irregular growth, periods of active elongation ranged from $\mathrm{I}$ to

Table I. Representative 'growth maps' selected from the $4 \mathrm{I}$ and 160 sections of the corresponding continuous growth tubes of the A-and B-cultures to illustrate the variation encountered in average daily growth prior to and following temporary cessation of growth in the adal-2 cultures maintained by continuous hyphal elongation

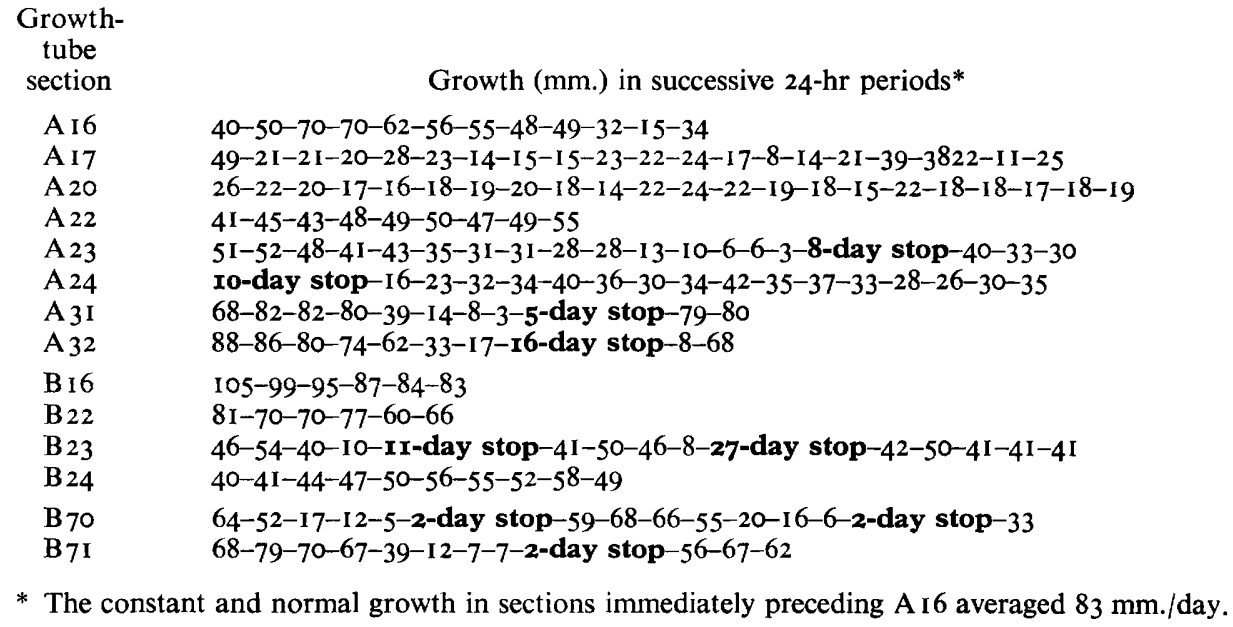

3I days, during which the hyphal tips advanced as little as $5 \mathrm{~mm}$. or as much as 1050 $\mathrm{mm}$. The periods of no measurable growth ranged from I day to over 3 months. During individual periods of active growth daily average rates ranged from 4 to $9 \mathrm{I}$ $\mathrm{mm}$./day; the latter rate was comparable to the maximum the culture attained in sections previous to section A I6. The culture stopped 48 times for a total of 780 days, and active growth was observed only during 285 days after the culture first stopped in A 23. The 48 stops in the final I 8 sections are evidence of the highly degenerative condition of the culture. It is impossible to estimate how long such aberrant growth would have continued had the culture not become contaminated; there is no reason to expect that it would not have continued after 3 years of stop-start growth. 
Observation of the A-culture showed that growth slowed considerably before stopping (Table I), and that during this period the mycelial frontier often was composed of only a few scattered hyphae. When it was possible to observe the resumption of hyphal elongation, new growth always started at points 10-I $40 \mathrm{~mm}$. proximal to the stopped frontier.

After stopping first occurred in A23, a growth tube of complete medium was connected to the proximal end of that section to see whether the enriched medium would affect growth. The culture stopped three times in this section of complete medium for 7, 29 and 27 days, compared with one 8-day stop in the original A23. Subsequent tests with complete medium at both $25^{\circ}$ and $35^{\circ}$ did not show any effect in decreasing either the number of times a culture stopped or the duration of the stops. In fact, complete medium usually more adversely affected growth than did minimal medium supplemented with adenine.

This unusual stop-start phenotype associated with the A-culture has been studied extensively in subcultures and heterokaryons. Its genetic basis was found to be an extranuclear factor, stp- $A$. The experimental evidence for these findings will be reported elsewhere.

\section{Morphological changes in the A-culture}

The decrease in growth rate in section A I 6 was accompanied by several changes in the morphology of the culture. For example, the production of conidia was greatly decreased in sections AI6 and AI7 and none could be detected microscopically in samples of mycelium removed from sections $\mathrm{A}_{3} \mathrm{I}$ to $\mathrm{A}_{4} \mathrm{I}$. This response appeared to be largely environmental, since many of the subcultures from these sections formed some conidia on agar slopes, but were again aconidial when grown in growth tubes. During the same period the A-culture also lost ability to function as a protoperithecial parent in crosses, and subsequent to AI6 all subcultures were female-sterile. The appearance of a brown pigment in ageing mycelium and a brown discoloration of the culture medium was another change which appeared in the culture after it had slowed down. Discoloration of the medium was particularly noticeable in regions where the culture ceased to grow temporarily.

\section{Cytoplasmic involvement in growth changes in the A-culture}

Following section A I 6 growth was very slow, but single conidial isolates from these sections showed a wide variation in growth rates. For example, the growth rate of the main culture in A 19 was less than $24 \mathrm{~mm}$./day, but single conidial isolates from the same section had rates ranging from 13 to $63 \mathrm{~mm}$./day and averaged $39 \mathrm{~mm}$./day. To determine whether these rates were due to nuclear or cytoplasmic heterogeneity, conidia from section A 19 were heterokaryotically combined $1: 9$ with conidia from a normal panal-I strain using the technique of Pittenger, Kimball \& Atwood (I955). This heterokaryon was assumed to have a high proportion of normal cytoplasm. It was predicted that adal-2 homokaryotic isolates recovered from the heterokaryon would have normal growth rates if the previously observed heterogeneity were due to the involvement of an abnormal cytoplasm but not if the heterogeneity were nuclear. From this heterokaryon, I 8 adal-2 homokaryons were recovered. Their growth rates ranged from 69 to $86 \mathrm{~mm}$./day and averaged $8 \mathrm{I} \mathrm{mm}$./day-a rate comparable to that 
found in early sections of the A-growth tube. Since such heterokaryotic rejuvenation indicated that the adal-2 nuclei were capable of normal growth in a normal cytoplasm, it appeared that the decreased growth rates of single conidial isolates from the Aculture resulted from extranuclear factors rather than nuclear factors.

Additional evidence for involvement of extranuclear factors was that panal-I homokaryons recovered from a heterokaryon between panal-I and adal-2 from section A 20 exhibited decreased growth rates. Normally, panal-I homokaryons grow at approximately I02 $\mathrm{mm}$./day. Of 33 panal-I homokaryotic isolates from the heterokaryon, five had growth rates of less than $48 \mathrm{~mm}$./day, two had stop-start growth phenotypes and the remainder had rates ranging between 48 and $9 \mathrm{r} \mathrm{mm}$./day. This was interpreted to mean that the integrity of the panal-r component, with respect to growth rate, had been altered by heterokaryotic association with the adal-2 strain. Presumably, the change was controlled by extranuclear factors.

\section{Description of growth of the B-culture of adal-2A}

The rapid decrease in growth rate in A I6 (Fig. I; Table I), which continued until A 23, where the culture temporarily stopped, suggested that some genetic change had taken place. Consequently, in addition to the usual procedure of adding a new section t's the distal end of A I6, one was also added to the proximal end; the mycelium, of course, was severed in separating section A I 5 from A I6. The mycelium in the proximal end of AI6 resumed growth immediately when brought in contact with the fresh medium. The new culture was subsequently called the B-culture. Because of its interesting phenotype it was followed from B 16 to B I60 when the experiment was terminated. Interestingly enough, both the A- and B-cultures continued to grow, although at very different rates, until sections A23 and B 23. There the respective cultures stopped temporarily for the first time after I4, I4O and I3,720 mm. of growth respectively.

In sections $\mathrm{B}_{1} 6$ to $\mathrm{B}_{19}$ the B-culture grew approximately $92 \mathrm{~mm}$./day, a rate well above the $58 \mathrm{~mm}$./day observed in the proximal half of AI6, but after B I9 a slow decrease in rate occurred within the subsequent $2000 \mathrm{~mm}$. until the culture grew only $48 \mathrm{~mm}$./day in the proximal half of B 23. Growth ceased in B 23 after nearly $4500 \mathrm{~mm}$. of growth in 55 days; it resumed I I days later and stopped again for 27 days after progressing only $145 \mathrm{~mm}$. in 4 days (Table $\mathrm{I}$ ). After growth resumed the second time, there were no significant increases in rate and one significant decrease (Fig. I) during the following $28,740 \mathrm{~mm}$. of growth in 519 days prior to B 70 . In section B 70 and continuing to B I 6 , there were four series of stops with each series consisting of from I to 8 stops. These series of stops and their duration are presented in Table 2. While the two initial stops of the culture were for I I and 27 days, the I 8 subsequent stops averaged slightly more than 2 days in duration and ranged from $I$ to 4 days. Although the stops in sections B 23 and B 70 occurred almost $1 \frac{1}{2}$ years apart with no intervening stops, the genetic determinant of stopping, stp- $B$, which Bertrand \& Pittenger (unpublished) have shown to be extranuclear in nature, appeared to be present, although not expressed, during the entire period. This evidence comes from isolating and growing conidia from subcultures of the intervening sections. Of 236 single colony isolates analysed from Io intervening sections, 66 , nearly $28 \%$, stopped during growth in $500 \mathrm{~mm}$. growth tubes.

Other major changes in rate that appeared during growth of the B-culture are shown 
in Fig. I. Several of the rate changes were accompanied by alterations in morphology. A description and genetic analysis of the off-types follows.

Table 2. A summary of the location and duration of the 20 temporary cessations of growth observed during 4 years of continuous hyphal propagation in the I6o sections of the continuous-growth tube of the B-culture, together with the number of days and $\mathrm{mm}$. of growth between the five series of stops

$\begin{array}{ccc}\begin{array}{c}\text { Growth-tube section in which } \\ \text { stopping occurred and distance } \\ \text { and days of growth between } \\ \text { series of stops }\end{array} & \begin{array}{c}\text { No. of times } \\ \text { culture stopped } \\ \text { in each } \\ \text { section }\end{array} & \begin{array}{c}\text { Duration of each } \\ \text { stop (days) }\end{array} \\ \begin{array}{c}\text { I3,720 mm. of growth in I 56 days } \\ \text { B 23 }\end{array} & 2 & \text { I I, 27 } \\ 28,740 \mathrm{~mm} \text {. of growth in 520 days } & 2 & 2,2 \\ \text { B 70 } & \text { I } & 2 \\ \text { B 71 } & 2 & 2,4 \\ \text { B 72 } & \text { I } & 3 \\ \text { B 73 } & 2 & 3,2 \\ \text { B 75 } & & 2 \\ \text { B } 86 & \text { I } & 2,1,1 \\ \text { B 88 } & 3 & 3 \\ \text { B } 89 & \text { I } & \\ 6300 \mathrm{~mm} \text {. of growth in } 98 \text { days } & & 3,3 \\ \text { B I04 } & 2 & 4,2 \\ \text { B I05 } & 2 & \text { I }\end{array}$

The variant-I phenotype

The decline in growth rate from 84 to $48 \mathrm{~mm}$./day in B I9 to B 23 was accompanied by marked alterations in morphology of the culture. For example, in B 23 the usual abundant production of aerial hyphae was decreased so the culture had a somewhat 'shaven' appearance and conidia were formed only sparsely. Likewise, functional protoperithecia were no longer formed when subcultures were grown on crossing medium. Irregularly shaped 'lysed' areas were also apparent in 2- to 3-week cultures. They were phenotypically recognized primarily by disappearance of aerial hyphae resulting in the appearance of clear areas. Lysis started only in aged mycelium as small discrete areas scattered throughout the length of a growth tube and progressively encompassed most of the surface as shown in Plate I. Although this trait is inherited, the biochemical mechanisms involved are not known.

The variant-I phenotype persisted in the B-culture from B 23 to B I60, suggesting that it resulted from a permanent genetic change. The phenotype was not subject to heterokaryotic rejuvenation, and segregated in crosses in which adal-2 var-I was used as a conidial parent. A total of 29 complete asci and 75 random spores from three crosses between $74 a$ and $a d a l-2$ var- $I$ from B 82 were analysed. Half of the ascospore cultures from each tetrad were var-I with respect to aerial growth, decreased conidiation, growth rate and female sterility. The progeny from six tetrads were checked for appearance of lysis. In all cases the cultures which showed the other properties of variant-I were also lytic. The variant-I phenotype segregated independently of mating 
type, requirement for adenine and albinism. These results are consistent with variant-I being controlled by a single gene mutation.

Apparently both the ad and var-I mutants individually decreased the growth rate and the effect was additive. This could be shown by growth rates of isolates from the above cross. The normal isolates from the cross averaged I I $\mathrm{mm} . / \mathrm{day}, 79$ individual ad strains averaged $85 \mathrm{~mm}$./day, 54 var- $I$ isolates averaged $69 \mathrm{~mm}$./day and 89 double-mutants had an average growth rate of only $45 \mathrm{~mm}$./day.

That the variant-I phenotype from various sections of the B-growth tube was the result of the same genotype was demonstrated as follows. An adal-2 var-I isolate from B I9 was crossed to a nic-Ial-288a strain and 2 I of 46 random spores isolated were phenotypically variant-I as expected if var-I were determined by a single genetic factor. A nic-Ial-2 var-IA culture with an average growth rate of $63 \mathrm{~mm}$./day was selected and heterokaryotically combined with adal-2 var-I subcultures from sections $\mathrm{B} 42,62,82, \mathrm{I} 20$ and $\mathrm{I} 40$. All the resultant heterokaryons, with growth rates averaging 6I $\mathrm{mm}$./day showed sparse conidiation, were female-sterile and showed lysis on ageing. This complete expression of the variant-I phenotype in all heterokaryons indicated genetic identity of the var-I genotype in the various sections of the Bculture.

Table 3. Neurospora crassa. Number of adal-2 and adal-2 var-1 single colony isolates from subcultures of various sections of $A$ - and B-growth tubes

$\begin{array}{cccc}\text { Section } & \begin{array}{c}\text { Total } \\ \text { isolates } \\ \text { tested }\end{array} & \overbrace{\text { adal-2 }}^{\text {Phenotypes of isolates* }} & \text { adal-2 var-I } \\ \text { A I3 } & 30 & 30 & 0 \\ \text { A 14 } & 30 & 30 & 0 \\ \text { A 15 } & 30 & 9 & 21 \\ \text { A 19 } & 20 & 20 & 0 \\ \text { B I6 } & 29 & 13 & 16 \\ \text { B 19 } & 30 & 3 & 27 \\ \text { B 20 } & 30 & 0 & 30 \\ \text { B 21 } & 30 & 0 & 20 \\ \text { B 23 } & 30 & 0 & 30\end{array}$

* Heterokaryons (adal-2 + adal-2 var-I) assumed to have adal-2 phenotype.

The presence in the A- and B-cultures of var-I nuclei long before the variant-I phenotype was expressed was demonstrated by isolating homokaryotic var-I conidia from subcultures of the two growth tubes. Homokaryotic adal-I var-I cultures could be distinguished easily from both heterokaryotic and adal-2 isolates by differences in conidiation in mature cultures on agar slants. The results in Table 3 show that only the adal-2 type was found in subcultures from sections $\mathrm{A} \mathrm{I}_{3}$ and A I 4 , but both adal-2 and adal-2 var-I isolates appeared in sections A I5, B I5, BI9 and B20. Finally, only adal-2 var-I homokaryons were obtained from subcultures of B $2 \mathrm{I}$ and B 23, and from all subsequent B-cultures. Since these determinations were made from subcultures and not directly from conidia of the B-culture, the proportions of the homokaryotic adal-2 var- $r$ isolates do not necessarily provide a good estimate of their frequency in the B-growth tube. Nevertheless the complete displacement of adal-2 by adal-2 var-I indicates a selective mechanism that favours var-I. 


\section{The variant-2 phenotype}

The second distinct change in morphology of the B-culture was observed at the same time that a significant change in growth rate occurred between sections B I5I and $B$ I 57 . The culture became aconidial in section B ${ }_{5} 6$ and aerial hyphae were largely absent by the end of BI57. Excessive hyphal branching in the growing tips of the culture was one of the characteristics noted. The phenotype of germinating conidia was also quite unique. One or two hyphae extended from the conidia and then branched extensively to give a fan-like layer of growth on the surface of the sorbose medium. When transferred to growth tubes, the cultures grew extremely slowly and never more than $7 \mathrm{~mm}$./day. The mycelium had the appearance of a flat thin rope with small lateral branches strung along the middle of the agar surface of the growth tube; there was no aerial growth and conidia were completely lacking. That this phenotype, called variant-2 (var-2 is the corresponding genotype), arose from adal-I var-I was suggested not only because it carried the same mutant genes, but also from crossing data.

Information available about the genetic basis of the variant-2 phenotype is limited, since this altered type gave extremely infertile crosses even when used as the male parent. Among cultures from I4 viable spores from a cross of $74 a$ with a single conidial isolate of variant-2, segregating for both $a d$ and $a l-2,8$ gave rise to cultures that were neither variant-I nor $-2,2$ were phenotypically like variant-I, and 4 had the variant-2 phenotype. Since we do not know whether variant-2 (presumably of the genotype var-I, var-2) can be distinguished from var-2 alone, we can only say that the variant-2 phenotype is heritable.

That var-2 was present in the B-culture before any morphological or growth-rate changes were observed was easily demonstrated. Conidia taken directly from B I 45 and plated formed two distinct types of colonies. About $96 \%$ were normal in size; the remaining $4 \%$ were extremely small and, when transferred to agar slope, had the phenotype described for variant-2. The proportion of such colonies, which represents the proportion of homokaryotic var-2 conidia and not var-2 nuclei, gradually increased to about $17 \%$ in BI50 through BI56 and then increased to $25 \%$ in BI57. Since conidia were absent from subsequent sections, no further estimates of the proportion of variant-2 nuclei are available. However, a comparison of the growth rate of variant2 homokaryons with the B-culture made it clear that the B-culture was still heterokaryotic through section B I6o where the experiment was terminated. Since the extremely decreased growth rate of var-2 compared with var-I was clearly nonadaptive, the increased proportion of var-2 nuclei was unexpected, and some selective mechanism must be postulated.

\section{DISCUSSION}

The growth rate achieved by a culture in a continous growth tube at any given time is determined by the interaction of genetic and environmental factors. It is obviously impossible in a culture with a decreased growth rate to assess which interacting factor limits growth rate unless one plays a dominant role that is reproducible in a subculture. Consequently, reproducibility of altered growth rates was a prerequisite in our attempts to determine whether spontaneous mutants were responsible for changes in growth rates of the A- and B-cultures. As expected, many significant increases and decreases 
in growth rates shown in Fig. I were not reproducible in subcultures. On the other hand, most of the reproducible rate changes observed in the A- and B-growth tubes not only had a genetic basis but also had the predicted suppressive phenotype. Although the completely different behaviour of the A- and B-cultures, which had a common origin through section A I6, emphasizes the problem of trying to generalize about growth behaviour of such continually growing cultures, both series acquired certain similar abnormal phenotypes which should be mentioned.

Both the A- and B-cultures, as well as several experimentally produced heterokaryons later studied (Grindle \& Pittenger, unpublished) during 3 or more months of continuous growth, were similar in that over long periods of time their growth rates were clearly non-adaptive. In all of these continuous cultures, significant increases in growth rate were short-lived and no evidence was found to indicate that they resulted from genetic alterations. However, since there are some genetic phenomena-for example, transient changes in nuclear or cytoplasmic proportions in heterokaryons and heteroplasmons-which might temporarily effect growth rates but would not be expected to be reproducible in subcultures, we can only speculate that the majority of non-reproducible rate changes were not caused by demonstrable genetic changes.

Clearly the most unusual aspect of the long-term growth study was that growth was not continuous but ceased repeatedly in both the A- and B-cultures, and resumed again in characteristically different ways. That is, the period of absence of growth was quite different in the two cultures, averaging slightly over 2 days for the B-culture and $\mathbf{I} 6$ days for the A-culture. The corresponding genetic determinants of stopping, $s t p-A$ and $s t p-B$, are cytoplasmically inherited (Bertrand \& Pittenger, unpublished).

It also seems clear that there were homeostatic mechanisms affecting growth rates, not only because of the general constancy of growth but also because every major decrease in rate was followed by an increase. Growth rates restored to values previously achieved were routinely noted after cultures had stopped temporarily, as well as in many cases of minor fluctuations. However, only rarely following a major decline was growth ever restored to the value observed before the decline.

One can also generalize that the naturally occurring genetic changes which took place during long-term vegetative growth of Neurospora resulted not only in an overall decrease in growth rate, but also an overall degeneration in both sexual and asexual reproductive capacity. For example, female sterility and decreased conidiation controlled by genetic factors occurred early in the growth of both A- and B-cultures. In this respect, our observations paralleled those reported by Jinks (1959) in Aspergillus except that in Neurospora the phenotypic changes were due to permanent genetic changes at both the cytoplasmic and nuclear levels. Jinks, on the other hand, ascribed them to cytoplasmic changes resulting from a readjustment in the equilibrium of normal cytoplasmic constituents. Furthermore, there was no indication in Neurospora for an irreversible cessation of growth as the final condition of degenerative processes associated with prolonged vegetative propagation, as observed for Aspergillus (Jinks, 1959) and Podospora (Marcou, I96r). In fact, Marcou's work with Podospora and other fungi suggested to her that indefinite hyphal propagation may be impossible in fungi. That generalization apparently does not apply to Neurospora.

The extranuclear determinants of the stopper A and B phenotypes, and the death of stopped hyphal tips, suggest that possibly these phenotypes and 'vegetative death' in Aspergillus and 'senescence' in Podospora are related. Occasional resumption of 
growth has been observed in dying cultures of Aspergillus, but the recoveries were short-lived and apparently did not occur repeatedly in the same culture (Jinks, 1959). That is not the case with Neurospora stopper mutants, where temporary cessations were the rule rather than the exception and recoveries were often long-lived. Consequently, if there is a relationship between the stopper phenotypes of Neurospora and irreversible cessation of growth in Aspergillus and Podospora, then Neurospora may have biological properties that permit it to survive deleterious cytoplasmic changes which are lethal in some other fungi. The extremely rapid growth of Neurospora compared with Podospora and Aspergillus immediately suggests that growth rate itself could be indirectly involved. That is, over extended periods of growth, the slower growing species may simply be unable to escape the unfavourable intracellular or extracellular environments that are a byproduct of their own growth. Similarly, the ability of Neurospora to resume growth might be nothing more than a simple difference among the organisms to invade media already occupied by their own dead mycelium. It is equally likely, however, that more subtle physiological phenomena are involved. It will be interesting to find out what biological properties are responsible for such differences between species of fungi.

We can also generalize to some extent about the unique properties of the detectable nuclear and extranuclear alterations that accumulated during growth. We have already referred to the fact that the unusual phenomena of stopping was associated with two extranuclear mutants. It was possible to demonstrate that the extranuclear determinants of stopping were present before stopping was first observed in the A- and Bcultures. This was done by isolating single conidial isolates from sections of the A-and B-growth tubes long before the cultures stopped in sections A 23 and B 23 and showing that some stopped in $500 \mathrm{~mm}$. tubes. The almost continual expression of the stopper phenotype in the A-culture plus the dominance of stp- $A$ in heterokaryons shows that this mutant has the property of suppressiveness predicted as characteristic of mutants expressed in continuously growing cultures. The same suppressiveness was manifested periodically for the $s t p-B$ extranuclear mutant. Although it stopped only periodically and the mutant could be demonstrated to be present 3 years after it was first observed, its behaviour is hardly suppressive in the way described for the stp- $A$ mutant.

Obviously, to see a culture stop growing gives no clue to the mechanism involved and we have no evidence to help us distinguish among several hypotheses. Two extreme possibilities are that either the culture 'runs out' of something essential to its growth like an energy synthesizing system, or it accumulates something like an abnormal structural protein (Woodward \& Munkres, 1966) that is detrimental to growth. Since the genetic basis of stopping is clearly extranuclear and because both stp- $A$ and $s t p-B$ have defective cytochrome- $c$ oxidase systems, it is assumed that the mitochondria are involved in some way. Other than this, however, we have no direct evidence for the mechanism which causes cessation or resumption of growth.

In addition to the cessation of growth associated with the extranuclear mutants, two other significant changes in growth rate deserve some consideration. Discussing only the B-culture, the presumptive suppressive phenotypes of the two nuclear mutants, $v a r-I$ and var-2, were of special interest. The complete displacement of the original adal-2 nuclear type by the adal-2 var-I seemed significant since the nuclear type with the highest selective value in a mixed population was the one with the slowest growth rate as a homokaryon (approximately 2.5 and $3.5 \mathrm{~mm}$./ hr for adal-2var-I and adal-2, 
respectively). It is also worth noting that the adal-2var-I type, which became established in the B-culture but not in the A-culture, was first present in A before B was even started in A I6. There was no evidence, however, for the persistence of var-I nuclei in the A-culture in sections distal to A23. The inability of var-I nuclei to become established in the A-culture is inconsistent with its postulated superior division rate, but it may be that the intracellular conditions of the A-culture were sufficiently different, due to the presence of extranuclear factors demonstrated to affect growth of the A-culture in A I9 and A20, that the var-I was unable to become established.

The var-2 mutant, which was first detected in B I 45, and was undoubtedly present much earlier in a lower frequency, also increased significantly during subsequent growth. Since the culture later failed to form conidia, we were unable to obtain further estimates of the proportion of var- 2 nuclei in the population. Nevertheless the $25 \%$ homokaryotic var-2 conidia found in B 157 indicated the proportion of var-2 nuclei in the heterokaryotic culture was considerably higher than $25 \%$ and possibly approached $50 \%$. Since the growth rate of var-2 homokaryons never exceeded $7 \mathrm{~mm}$./ day, the only two nuclear mutants isolated that affected growth both had significantly lower homokaryotic growth rates than the nuclear types simultaneously present in the B-culture. Despite the decreased homokaryotic growth rates, var-I and var-2 had selective advantages in heterokaryons as measured by their increase in the population during growth. The simpler explanation of such selection, which was clearly non-adaptive in terms of growth rate, is that it was achieved as a result of an increased division rate of the mutant types; controlled reconstruction experiments have not yet been done. Since this behaviour phenotypically is suppressive, the similarity of these nuclear mutants to the suppressive extranuclear mutants, stp- $A$ and $s t p-B$, is obvious. Var- $I$ and $s t p-A$ were similar in that both apparently caused a nearly complete displacement of the corresponding nuclear and extranuclear homologues of the culture. The analysis of var-2 was not complete enough to determine whether it would have eventually displaced var- $I$. The general significance of the observed increases in the proportion of slower-growing components of heterokaryons is unknown. While such slow-growing types would be rapidly eliminated in a population of uninucleate cells, they appear to have some selective value in a coenocytic heterokaryotic mycelium. Grindle \& Pittenger (unpublished) have also observed that in a number of experimental heterokaryons there was a significant increase in the proportion of the slower-growing nuclear component during extended periods of growth, this phenomenon would not appear to be completely unique.

This publication is Contribution no. 985, Department of Agronomy, Kansas Agricultural Experiment Station, Manhattan, Kansas, U.S.A. The work was supported by NSF grant no. GB2I00.

\section{REFERENCES}

JINks, J. L. (1958). Cytoplasmic differentiation in fungi. Proc. R. Soc. B r48, 3 I4.

J1NKS, J. L .(1959). Lethal suppressive cytoplasms in aged clones of Aspergillus glaucus. J. gen. Microbiol. 21, 397.

JINKs, J. L. (I964). Extrachromosomal Inheritance. Englewood Cliffs, N.J.: Prentice-Hall.

MarCou, D. (1961). Notion de longévité et nature cytoplasmique du déterminant de la sénescence chez quelques champignons. Annls Sci. nat. (Bot.) series I 2, p. 653.

McDougall, K. J. \& Pittenger, T. H. (I962). Observations of perpetual hyphal propagation in Neurospora. Neurospora News 2, 10. 
McDougall, K. J. \& Pittenger, T. H. (1966). A cytoplasmic variant of Neurospora crassa. Genetics $54,551$.

Pittenger, T. H., Kimball, A. W. \& Atwood, K. C. (1955). Control of nuclear ratios in Neurospora heterokaryons. Am. J. Bot. 42, 954.

Ryan, F. J., Beadle, G. W. \& Tatum, E. L. (1943). The tube method of measuring the growth rate of Neurospora. Am.J. Bot. 30, 784.

VoGel, H. J. (1956). A convenient medium for Neurospora (medium N). Microbiol. Genet. Bull. 13, 42.

WestergaArd, M. \& Mitchell, H. K. (I947). Neurospora. V. A synthetic medium favouring sexual reproduction. Am. J. Bot. 34, 573.

WOODWARD, D. O. \& MUNKRES, K. D. (1966). Alterations in a maternally inherited mitochondrial structural protein in respiratory deficient strains of Neurospora. Proc. Natn. Acad. Sci. U.S.A. 55,872 .

\section{EXPLANATION OF PLATE}

The upper photograph (actual size) of an adal-2A strain of Neurospora crassa in a continuousgrowth tube shows the appearance of the initial stage of 'lysis' which encompasses a relatively small area. The culture had been growing continuously for over 2 years. The two lower photographs, at slightly higher magnifications, show two areas from the same growth tube as the upper photograph after 'lysis' had progressed throughout the culture during the following week. Note the small remaining white patches of 'unlysed' mycelium. The vertical structure in the middle left of the upper photograph and at the right in the two lower ones is a cotton-stoppered sampling port of the growth tube. 

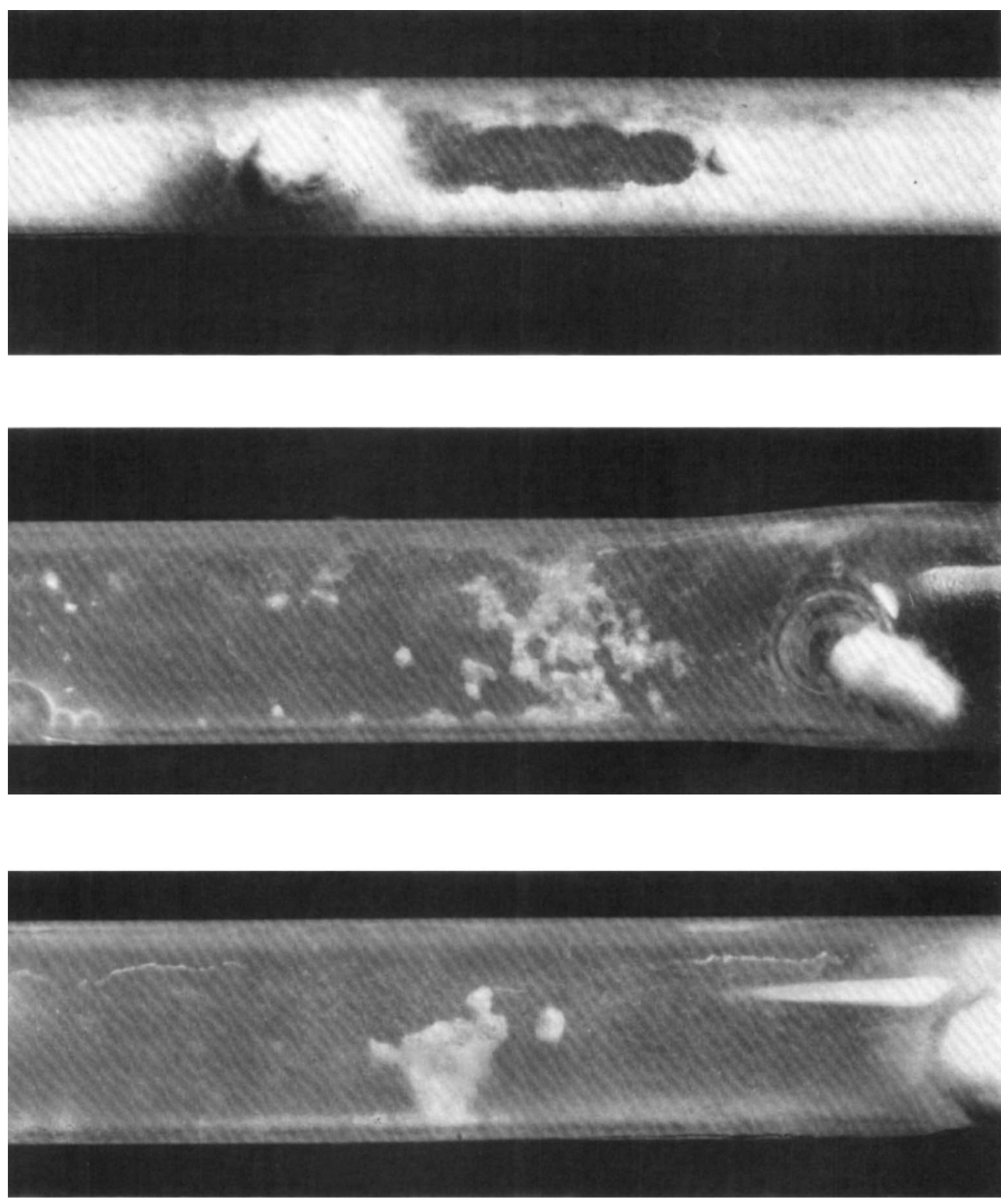